\title{
Evaluation of antidiabetic efficacy of polyherbal formulations in experimentally induced hyperglycemic rats
}

\author{
R. Duraisami*, S. Sengottuvelu, T. Prabha, S. Sabbani, S. Divya Presenna* and C.K. Muralitharan** \\ Department of Pharmacognosy, Nandha College of Pharmacy, Erode-638052, Tamilnadu, India \\ *Nandha College of Nursing, Erode-638052, Tamilnadu, India \\ **Nandha College of Physiotherapy, Erode-638052, Tamilnadu, India
}

\section{Article Info \\ Article history \\ Received 16 October 2021 \\ Revised 7 December 2021 \\ Accepted 9 December 2021 \\ Published Online 30 December 2021}

Keywords

Polyherbal formulations

Antidiabetic

Secondary metabolites

Blood glucose

\begin{abstract}
Polyherbal formulations, as the name suggests, include numerous components of various herbal origins. The plant secondary metabolites might have a broad range of pharmacological activities. Polyherbal formulations are primarily used to boost the activity of chemicals derived from other plants or to prevent their harmful effects. Owing to the inclusion of numerous secondary metabolites, these formulations may have a synergistic, potentiated, agonistic/antagonistic pharmacological impact. These mixtures contain numerous active ingredients with varied mechanisms of action that can work together to combat various diabetic problems. The focus of this research is to create a polyherbal formulation and test its antidiabetic properties in animal models. The study results showed the acute toxicity trials of the polyherbal formulation at dosages up to $2000 \mathrm{mg} / \mathrm{kg}$ over 14 days revealed no adverse effects. The crude extract of polyherbal formulation at $200 \mathrm{mg} / \mathrm{kg}$ lowers the fasting blood glucose levels however, the higher dose, $400 \mathrm{mg} / \mathrm{kg}$, lowers the blood glucose levels, significantly when compared to diabetic control groups. Whereas, the decoction of polyherbal formulation at $200 \mathrm{mg} / \mathrm{kg}$ produced a less significant reduction and the higher dose at $400 \mathrm{mg} / \mathrm{kg}$ produced a significant reduction in blood glucose level. Besides, the powder of polyherbal formulation at a high dose $(400 \mathrm{mg} / \mathrm{kg})$ produced a less significant reduction in blood glucose level on the seventh day, when the lower dose did not show any effect on the blood glucose level.
\end{abstract}

\section{Introduction}

Diabetes mellitus (DM) is a chronic condition due to an underlying or acquired deficiency in insulin levels by the pancreas or by the lack of effectiveness of insulin production. This insufficiency results in increased blood glucose levels that cause damage the many organ functions, particularly the blood vessels and nerves (Syed et al., 2005). The percentage of diabetes is increasing rapidly worldwide. The World Health Organization (WHO) has foretold that the number of adults with diabetes by 2030 will have almost doubled worldwide, from 177 million in 2000 to 370 million. The major causes of death in diabetes are cardiovascular diseases like atherosclerosis. A marked geographical variation in the prevalence of type 2 diabetes exists the highest rates are found in Americans of Arizona and in the inhabitants of the south pacific island of Nauru, where half the adult population has diabetes. In the rural communities of China and Chile, the prevalence is less than $1 \%$. The rates of type II DM are higher in urban populations than in rural communities. Type I DM accounts for up to the $10 \%$ of all cases of diabetes is rising. Across Europe, the average annual increase in the incidence in children under 15 years old is $3.4 \%$, with a steep rise in those under 5 years old. Type II DM accounts for around $90 \%$ of all cases of diabetes. There are approximately 1.4 million people with diagnosed type II DM.

Corresponding author: Dr. R. Duraisami

Professor and Head, Department of Pharmacognosy, Nandha College of Pharmacy, Perundurai Main Road, Erode-638052, T.M., India E-mail:duraisamirasilingam@gmail,com Tel.: +91-9842787144

Copyright (c) 2021 Ukaaz Publications. All rights reserved.

Email: ukaaz@yahoo.com; Website: www.ukaazpublications.com incidence of diabetes increases with age, with most cases being diagnosed after the age of 40 years (Shipra et al., 2009).

The heritability of type II DM is nearer than for type I DM, and is estimated to account for $40-80 \%$ of total disease susceptibility many patients will have a family history of diabetes, and twin studies show a high concordance rate (60-90\%) in monozygotic twins the maternal history of diabetes confers a higher risk of type II DM in the offspring than paternal history. Overweight and lack of physical activity are the two most major environmental risk factors for diabetes. It is estimated that up to $80 \%$ of all new cases of diabetes can be contributed to obesity in the UK. The average body mass index (BMI) of a person with type II diabetes is $30.0 \mathrm{~kg} / \mathrm{m}^{2}$; in the USA, $67 \%$ of more than $27 \mathrm{~kg} / \mathrm{m}^{2}$ and $46 \%$ have a BMI of more than $30 \mathrm{~kg} / \mathrm{m}^{2}$. The risk of developing type II increases across the normal range of BMI, such that the risk in the middle ages women whose BMI is over $35 \mathrm{mg} / \mathrm{m}^{2}$ is 93.2 times greater than in a woman whose BMI is below $22.5 \mathrm{~kg} / \mathrm{m}^{2}$ (Shipra et al., 2009).

In worldwide, only India having the highest number of diabetic patients, the disease is becoming an enormous health problem in the country; due to this reason now India calling as "the capital of the diabetic world". The International Diabetic Federation (IDF) was predicted that the number of patients with diabetes mellitus in India more than double from 19 million in 1995 to 40.9 million in 2007. It is estimated by 2025 will increase to 69.9 million. At present, in India up to $3 \%$ of the rural population and $11 \%$ of the city population above the age of 15 has diabetes. The WHO estimated that mortality from diabetes and heart disease costs India about 210 billion dollars every year and is expected to increase by 335 Billion Dollars in the next 10 years (Singh et al., 2003). 
In diabetes, the major complication is hyperglycemia and hyperlipidemia. Hyperglycemia is characterized by elevated levels of blood glucose, this leads to the generation of secondary complications like atherosclerosis, hyperosmolar nonketotic coma, diabetic nephropathy, diabetic retinopathy, and diabetic neuropathy as well as the hyperlipidemia is characterized by elevated levels of cholesterol, triglycerides, phospholipids, and changes in lipoprotein composition. The most common lipid abnormality in diabetes is hypertriglyceridemia, which is associated with metabolic consequences of coagulability, hyperinsulinemia, insulin resistance, and insulin intolerance (Rang et al., 2006).

Around the world and especially in developing countries, about $80 \%$ of the people have been using herbal remedies. The herbal products are thought to be less harmful, safer, and more culturally acceptable, as well as having more effectiveness, strength, and fewer side effects (Latha et al., 2021). According to the WHO report, over 21000 plant resources have been used for medical purposes across the world. Herbal remedies are largely has been using by Indian people. Its climate is very favorable to the growth of medicinal plants, around 45000 plant species, of which 15000 plants are flowering plants having about 1000 species identified as medicinal plants (Divya and Sanjeev, 2021). Due to this reason, India is also called the "Medicinal Garden of The World". Only 40 plant species are currently used by pharmaceutical companies (Mohamed et al., 2006).

Several treatments have been available for alternative therapy of diabetes. These can decrease the hyperglycemic condition but not identify the exact mechanism of action. Antioxidants relatively have the potential to inhibit the oxidation chain reactions at even small concentrations also. Several plant antioxidants are experimentally proved and used as effective protective agents against oxidative stress. It has been recognized that the antihyperglycemic effect of these plants either may be due to stimulation of pancreatic $\beta$ cells or the facilitation of metabolite in insulin-dependent process or inhibit the intestinal absorption of glucose (Mahendran et al., 2014; Akanksha et al., 2010).

\section{Materials and Methods}

\subsection{Plant collection and treatment}

The plants were collected in Vaikaalmedu and authenticated in the Department of Pharmacognosy, Nandha College of Pharmacy, Erode, Tamilnadu. The collected plant material was washed in running water, shade dried, and pulverized to get the powdered drug. The material was subjected to further experimental purposes.

\subsection{Experimental animals}

Swiss Albino mice and Wistar rats were purchased from The Animal House, Department of Pharmacology, IRT Perundurai Medical College, Erode, Tamilnadu. Animals were housed in the Animal House, Nandha College of Pharmacy and Research Institute, Erode, Tamilnadu. The animals were placed randomly in polypropylene cages with paddy husk as bedding and housed at standard condition maintained a temperature of $24 \pm 2^{\circ} \mathrm{C}$ and relative humidity of 30$70 \%$. A $12 \mathrm{~h}$ light and dark cycles were strictly followed throughout the study. The animals had free access to a standard animal pellet diet (Sukumar Agro Industries, Pune) and water. The Animal Ethical Committee of Nandha College of Pharmacy, Erode, Tamilnadu, approved our research proposal and presented it by CPCSEA guidelines.

\subsection{Chemicals and drugs}

The chemicals and drugs were purchased as LR and AR grade, those were alloxan monohydrate (Sigma Chemicals, Bangalore), formalin solution (Nice Chemicals, Cochin), carboxymethylcellulose, and Diethyl ether (Nice Chemicals, Cochin).

\subsection{Preparation of polyherbal formulations}

The composition and ratio of herbal ingredients are selected according to the potency of antidiabetic activity, which was stated in previous references, those were powder form of polyherbal formulation, decoction of polyherbal formulation, and crude extract of polyherbal formulation.

\section{i. Preparation of powder of polyherbal formulation (PPHF)}

The coarsely powdered plant materials were sieved through sieve no. 60. It helps to get the uniform-sized powder and according to the weight of individual powder, which is present in Table 1 has taken into a beaker and mixed well by using a suitable blender to get homogenous powder formulation. Then it was packed in a suitable container and kept in a dry place.

Table 1: Composition of polyherbal formulation

\begin{tabular}{|c|l|c|}
\hline S.No. & Plant name & Weight percentage $\mathbf{( \% )}$ \\
\hline 1 & Aegle marmelos & 2.66 \\
2 & Annona squamosa & 9.32 \\
3 & Bougain villia & 2.66 \\
4 & Cassia auriculata & 6.66 \\
5 & Emblica officinale & 8.00 \\
6 & Ficus carica & 13.30 \\
7 & Hibiscus rosa-sinensis & 6.66 \\
8 & Psidium guajava & 6.66 \\
9 & Stevia rebaudiana & 9.32 \\
10 & Tea leaves & 2.66 \\
11 & Tenospora cardifolia & 10.66 \\
12 & Terminalia chebula & 8.00 \\
13 & Zingiber officinale & 13.30 \\
\hline
\end{tabular}

\section{ii. Preparation of decoction of polyherbal Formulation (DPHF)}

All individual powders were taken in a beaker and mixed well with a suitable blender until to get a uniform powder formulation. Then packed the formulation in water diffusible paper to make easily dip in the hot water for making of decoction.

\section{iii. Crude extract of polyherbal formulation (EPHF)}

The polyherbal formulation is a mixture of all the individual different parts of plant extracts. It contains Aegle marmelous (leaves), Annona squamosa (leaves), Bougan villia (leaves), Cassia auriculata (flowers), Emblica officinale (fruits), Ficus carica (fruits), Hibiscus rosa-sinensis (flowers), Psidium guajava (leaves), Stevia rhubidiana (leaves), Camellia sinensis, Tenospora cardifolia (leaves), Terminalia chebula (fruit), Zingiber officinale (rhizome). 
This extraction is carried out by the maceration process for $24 \mathrm{~h}$. All the thirteen dried and pulverized plant ingredients were taken in individual conical flasks. Then added some amount of water up to rinse the powder, gently agitated for the first $6 \mathrm{~h}$, and kept aside for the remaining $18 \mathrm{~h}$. After $18 \mathrm{~h}$, filtered them individually, finally evaporated the solvent at $100^{\circ} \mathrm{C}$ up to get a paste-like preparation. Then, the different weights of the extracts, according to Table 1, taken into a beaker, mixed them properly with a little amount of water, and evaporated the water up to get a paste-like preparation and kept in the refrigerator after being packed in a suitable container (Sengottuvelu et al., 2008).

\subsection{Pharmacological study}

\subsubsection{Acute oral toxicity study}

Acute oral toxicity refers to those adverse effects occurring due to oral administration of a single dose or multiple doses are given within $24 \mathrm{~h}$. For every phase, three animals were employed, and the beginning dose is chosen from among the four predetermined values of 300,1000 , and $2000 \mathrm{mg} / \mathrm{kg}$ body weight. The use of the increased upper dosage level of $5000 \mathrm{mg} / \mathrm{kg}$ body weight may be explored in rare situations and only when warranted by special regulatory reasons.

\subsubsection{Acute toxicity studies}

Three mice (25-30 gm) were maintained under standard laboratory conditions. A total of 3 animals have to be used, which received a single oral dose of $2000 \mathrm{mg} / \mathrm{kg}$ body weight of polyherbal formulation. Animals were kept overnight fasting before the drug administration. After the administration of 3 doses of 3 formulations (extract, decoction, and powder) of the polyherbal formulation, food should be held for a further 3-4 h. Animals have to be observed individually once during the first $30 \mathrm{~min}$ after the dose given, predominantly during the first $24 \mathrm{~h}$ and daily for 14 days. Daily, once cage-side observations have to be done to find out the changes in skin, eyes, mucous membrane (nasal), respiratory rate, circulatory (heart rate and BP), anatomically (salivation, lacrimation, perspiration, urinary incontinence, and defecation) and central nervous system (ptosis, drowsiness, gait, tremors, and convulsions) changes.

\subsubsection{Screening of antihyperglycemic activity}

Healthy Wistar albino rats of either sex weighing 150-250 gm were used and before initiating the experiment, the animals were acclimatized in laboratory condition for seven days. The animals were kept in polypropylene cages, provided with a standard animal pellet diet (Pranav Agro Industries, Pune) and water and libitum. The animals were divided into nine groups of six each after overnight fasting all of the animals, except the normal control group, were injected with alloxan monohydrate suspension intraperitoneally, at a dose of $150 \mathrm{mg} / \mathrm{kg}$ body weight. The animals were allowed to stay for $72 \mathrm{~h}$ provided and diabetes was conformed, if the blood glucose levels exceed $200 \mathrm{mg} / \mathrm{dl}$ of blood. Drug doses were prepared by suspending the appropriate quantity of drug in $0.5 \% \mathrm{w} / \mathrm{v}$ of carboxymethylcellulose solution. The control animals were treated with $2 \mathrm{ml}$ of vehicle daily through the oral route and the all other groups were daily orally administered with their corresponding dose levels according to their body weight for 7 days as follows.

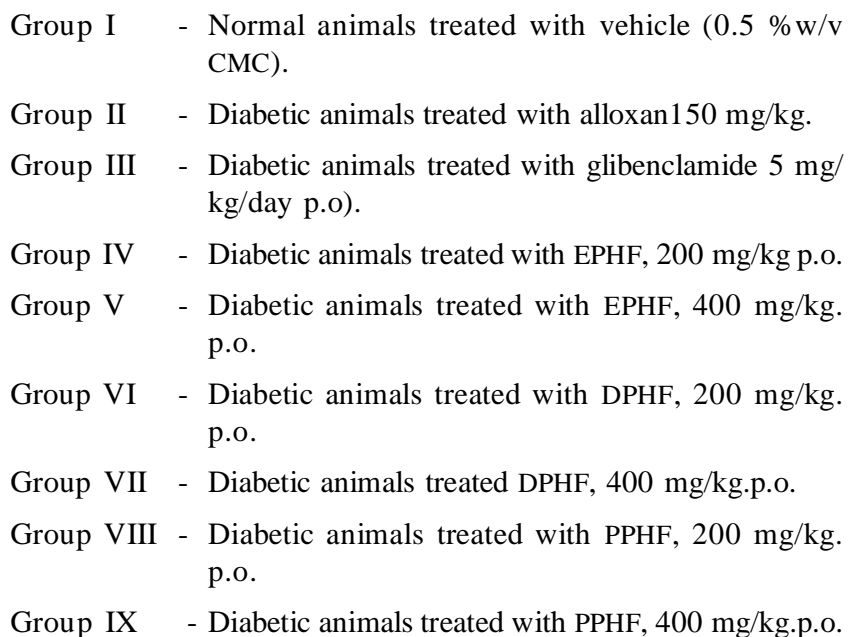

The animals fasting blood sugar levels were measured using a glucometer (Model- Accucheck, Roche Germany) after 1, 3, 5, and 7 days of treatment plans. Blood was collected from the tail tip of the animals after $3 \mathrm{~h}$ of drug administration, and the tail wound is applied with povidone-iodine ointment to prevent any infection. The fasting blood glucose levels in various polyherbal formulations treated animals were compared with that of diabetic control group animals (Bhise et al., 2009; Chandra et al., 2007).

\subsection{Statistical analysis}

The data pertaining to proper statistical testing, including one-way ANOVA (Analysis of Variance) and a Dunnett's post hoc test. Less significant, significant, and more significant were defined as $p>0.05$, $p>0.01$ and $p>0.001$, correspondingly. Graph pad prism software, version 4 , was used to do the study.

\section{Results}

\subsection{Acute oral toxicity of polyherbal formulations in mice}

In Table 2, the represented data indicates the changes in behavioral and physiological activities of mice with different doses of polyherbal formulation. The mice in the acute toxicity study daily received polyherbal formulations at dose levels of 300,1000, and $2000 \mathrm{mg} / \mathrm{kg}$. The duration of this study is 14 days. Observed behavioral changes in mice with polyherbal formulations are given in Table 2. By comparing with the control group, it was observed that extract of polyherbal formulation at doses 1000 and $2000 \mathrm{mg} /$ $\mathrm{kg}$ showed drowsiness. Except this, it was not shown any effect on the function of kidney, CNS, Liver, gastrointestinal tract, respiratory system, and color of skin, so no behavioral changes and no mortality was observed in mice with polyherbal formulations.

\subsection{Result of antihyperglycemic activity}

The effects of polyherbal formulations (EPHF, DPHF, and PPHF) on fasting blood glucose levels in alloxan-induced diabetic rats were given in Table 3 and Figure 1. On the seventh day of the treatment protocol, the lesser dose of both EPHF and DPHF at $200 \mathrm{mg} / \mathrm{kg}$ lowers the fasting blood glucose levels, whereas there was a significant reduction in blood glucose level observed at $400 \mathrm{mg} / \mathrm{kg}$ when compared to diabetic control groups. However, the PPHF at a high dose at $400 \mathrm{mg} / \mathrm{kg}$ only produced a decreased reduction in blood glucose level. 
Table 2: Results for acute oral toxicity study of polyherbal formulation

\begin{tabular}{|c|l|c|c|c|c|c|c|c|c|}
\hline Group & Treatment & Dose $(\mathbf{m g} / \mathbf{k g})$ & Salivation & Lacremation & Mucous & Urinary & Defecation & Tremors & Drowsiness \\
\hline I & CMC & $1 \mathrm{ml}$ & - & - & - & - & - & - & - \\
II & Extract & 300 & - & - & - & - & - & - & - \\
III & Extract & 1000 & - & - & - & - & - & - & + \\
IV & Extract & 2000 & - & - & - & - & - & - & + \\
V & Powder & 300 & - & - & - & - & - & - & - \\
VI & Powder & 1000 & - & - & - & - & - & - & - \\
VII & Powder & 2000 & - & - & - & - & - & - & - \\
VIII & Decoction & 300 & - & - & - & - & - & - & - \\
IX & Decoction & 1000 & - & - & - & - & - & - & - \\
X & Decoction & 2000 & - & - & - & - & - & - & - \\
\hline
\end{tabular}

Table 3: The effect of different polyherbal formulations on fasting blood glucose level

\begin{tabular}{|c|l|c|c|c|c|}
\hline Group & Treatment $(\mathbf{m g} / \mathbf{k g})$ & $\mathbf{1}^{\text {st }} \mathbf{d a y}$ & $\mathbf{3}^{\text {rd }}$ day & \multicolumn{1}{|c|}{$\mathbf{5}^{\text {th }}$ day } & $7^{\text {th }}$ day \\
\hline I & Normal control (CMC-0.5\%) & $081.5 \pm 1.15$ & $81.6 \pm 1.20$ & $82.6 \pm 1.70$ & $83.4 \pm 0.90$ \\
II & Diabetic control (alloxan-120) & $211.7 \pm 3.50 * *$ & $237.7 \pm 2.90 * *$ & $249.4 \pm 3.80 * *$ & $268.7 \pm 3.20 * *$ \\
III & Positive control (glibenclamide-5) & $206.4 \pm 1.70$ & $177.6 \pm 2.06 * *$ & $137.2 \pm 0.88^{* *}$ & $109.5 \pm 0.50 * *$ \\
IV & EPHF (200) & $197.5 \pm 2.90$ & $186 \pm 0.50 *$ & $165.3 \pm 2.00 *$ & $152.3 \pm 1.70 *$ \\
V & EPHF (400) & $206.5 \pm 3.05$ & $182 \pm 1.50 *$ & $152.5 \pm 2.00 * *$ & $126.8 \pm 1.70 * *$ \\
VI & DPHF (200) & $208.6 \pm 5.30$ & $192.3 \pm 1.30$ & $173.3 \pm 2.08$ & $165.7 \pm 1.10^{*}$ \\
VII & DPHF (400) & $218.3 \pm 0.80$ & $184.6 \pm 1.30$ & $161.3 \pm 1.40 *$ & $149.6 \pm 1.70 * *$ \\
VIII & PPHF (200) & $214 \pm 2.60$ & $204.1 \pm 3.20$ & $188.3 \pm 2.30$ & $175.2 \pm 2.00$ \\
IX & PPHF (400) & $221.6 \pm 3.40$ & $205.3 \pm 2.10$ & $174.6 \pm 2.18$ & $168.3 \pm 5.30 *$ \\
\hline
\end{tabular}

All values are mean \pm SEM, $n=3$. One-way Analysis of Variance (ANOVA), followed by Dunnett's test was performed as the test of significance.

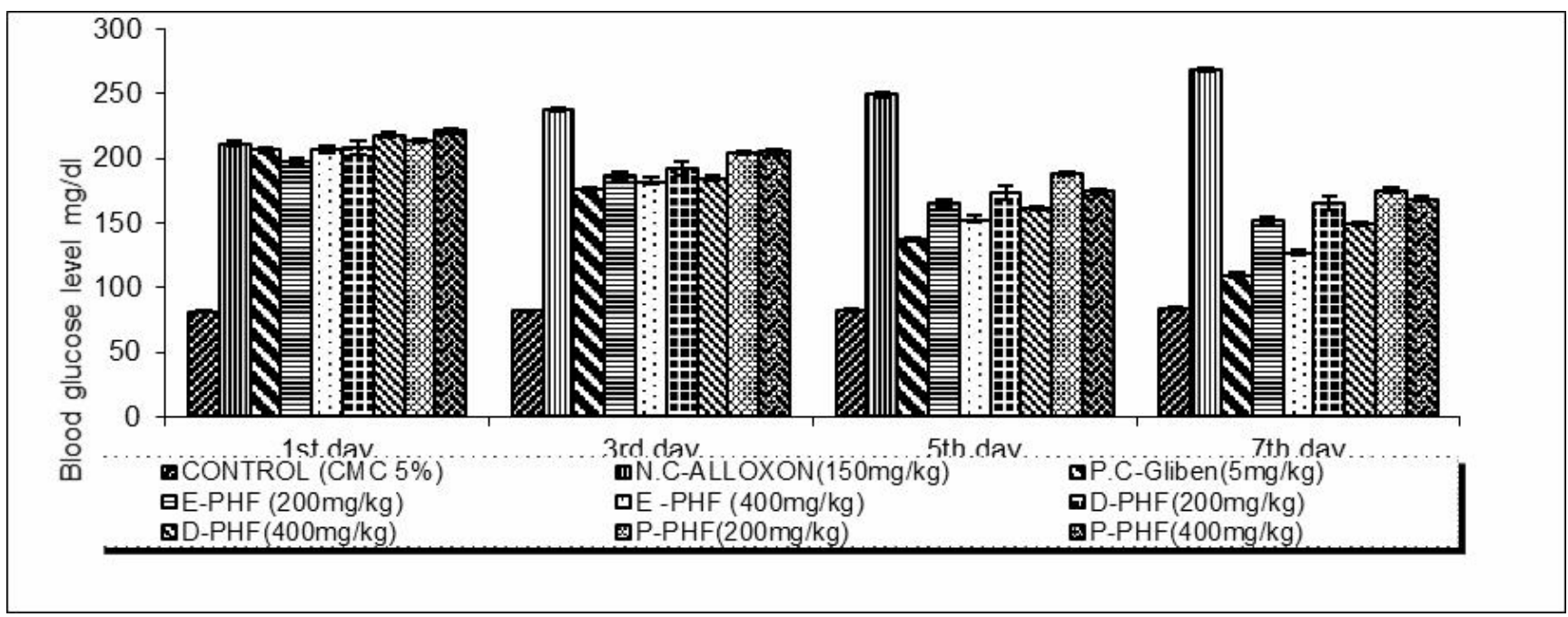

Figure 1: The fasting blood glucose level after the treatment protocol of polyherbal formulation in diabetic rats.

\section{Discussion}

Diabetes mellitus (DM) is a prevalent endocrine illness that affects more than 150 million people globally, with that figure expected to rise to 300 million by 2025 , out of which the Indians are accounting for more than one-fifth of those affected. India has been designated as the world's diabetes center by the International Diabetes Federation (Satyanarayana et al., 2010). Synthetic drug therapies for diabetes are now developed however, the prolonged use of those drugs could cause greater side effects to lead to death. Hypoglycemic episodes, nausea, dermatitis, diarrheas', jaundice, vision abnormalities, anemia, 
and other side effects have been reported with glibenclamide, metformin, repaglinide, and pioglitazone, among others. Polyherbal treatments contain synergistic, potentiating, agonistic, and antagonistic pharmacological substances that operate in harmony to generate treatment effectiveness with minimal adverse effects (Nupur, 2021).

The previous studies for the treatment of diabetes with polyherbal formulation exert good acceptable results (Petchi et al., 2014; Kaleem et al., 2008). The polyherbal formulation is assessed in diabetic rats at $500 \mathrm{mg} / \mathrm{kg}$ showed its effectiveness in oral glucose tolerance test and antidiabetic activity, but it does not produce a hypoglycemic effect. The efficacy of polyherbal formulation in obesity-related diabetes was studied by patil et al. (2010). The antidiabetic and antiobesity effect of the formulation was found to be nearly similar to that observed for glibenclamide and sibutramine, respectively.

The toxicological evaluation of polyherbal formulation is useful to evaluate its toxic effects which were produced on long-term administration. In the acute toxicity study of polyherbal formulations, mortality was observed at any dose. At dose levels of 1000 and 2000 $\mathrm{mg} / \mathrm{kg}$ P.O. of extract of polyherbal formulation only produces drowsiness, except this, no behavioral changes were observed at different doses of polyherbal formulations. The symptoms which were observed in acute toxicity represent the safety and no toxicity of polyherbal formulation (Sabu et al., 2009).

Alloxan induces a wide variety of animal species by damaging the insulin-secreting pancreatic $\beta$-cell, resulting in a decrease in endogenous insulin release, which paves the way for the decreased utilization of glucose by the tissues (Choudhury et al., 2017). $\beta$ cells' destruction is happened by the free radicals, which are generated in the fenton reaction of alloxan metabolism. The formation of hydroxyl radicals ( $\mathrm{OH}-$ ) from the hydrogen peroxide $\mathrm{H}_{2} \mathrm{O}_{2}$ is called fenton. Due to this, blood glucose levels of rats were increased significantly. Glibenclamide is a standard drug that will significantly decrease the raised blood glucose levels. In $\beta$-cells of the pancreas, $\mathrm{Na}+$ ion channels are responsible for depolarization which will increase the production of insulin and $\mathrm{K}^{+}$ion channels are responsible for repolarisation which will decrease the production of insulin. Glibenclamide increases the production of insulin by inhibiting the $\mathrm{K}^{+}$ion channels (Punith et al., 2019).

In the present experiment, administration of polyherbal formulations for seven days prevented a significant elevation of glucose levels in diabetic rats. This could be due to the result of improved glycemic control produced by the formulation. When related to other dosages of decoction and powder formulation, the extract of polyherbal formulation at a dose of $400 \mathrm{mg} / \mathrm{kg}$ P.O showed a substantial drop in fasting blood glucose levels. The pronounced antihyperglycemic effect may be because of the synergistic effect of various active principles in the ingredients of polyherbal formulation. The reduced fasting blood glucose level may be either due to the increase in glycogenesis, decrease in glycogenolysis, or increase in the entry of glucose molecules to various skeletal muscles (Petchi et al., 2014).

\section{Conclusion}

Diabetes is a chronic clinical syndrome little is talked about in aspects of prevention and curation, but rather the management, there is an increased focus on herbal medicines in the search for appropriate hypoglycemic and hyperglycemic agents. Many medicinal plants are effective in diabetes mellitus; acute toxicity study helps to improve the acceptability and authenticity of the herbal plants. The polyherbal formulation consists of thirteen plant-origin ingredients, which are individually used traditionally in the treatment of diabetes mellitus. Each plant act by different mechanisms to treat diabetes.

Based on the above results, it could be concluded that extract of polyherbal formulation exerts significant hypoglycemic and antioxidant activities. When compared to extract formulation, the decoction of polyherbal formulation and powder formulation had less potency on diabetic activity. Due to hyperglycemia, several chronic secondary complications will generate like diabetic ketoacidosis, diabetic retinopathy, diabetic neuropathy, diabetic nephropathy, and hyperosmolar nonketotic coma. The polyherbal formulation has capable of control of hyperglycemia. As a result, it may be concluded that polyherbal formulation treats diabetes' secondary problems gradually. Continued research is needed to determine the actual underlying mechanisms of antidiabetic efficacy of polyherbal formulation, as well as the involvement of the exact active secondary metabolites present in it.

\section{Conflict of interest}

The authors declare no conflicts of interest relevant to this article.

\section{References}

Akanksha, Aravind, K.S. and Rakesh, M. (2010). Antihyperglycemic activity of compounds from Indian medicinal plants. Indian Journal of Experimental Biology, 48:294-298.

Bhise, S.B.; Salunkhe, V.R. and Wachasundar, N.P. (2009). Clinical evaluation of antidiabetic polyherbal ayurvedic formulation: Madhujeevan churna using Stevia rebaudiana Indian J. Pharm. Pract., 2(2):2009.

Chandra, S.J.; Ekambaram, S.P. and Subramanian, V. (2007). Hypoglycemic and antilipidperoxidative effects of a polyherbal formulation, Diakyur, in experimental animal models. Journal of Health Sciences, 53(6):734-739.

Choudhury, H.; Pandey, M.; Hua, C. K.; Mun, C. S.; Jing, J. K.; Kong, L.; Ern, L. Y.; Ashraf, N. A.; Kit, S. W.; Yee, T. S.; Pichika, M. R.; Gorain, B. and Kesharwani, P. (2017). An update on natural compounds in the remedy of diabetes mellitus: A systematic review. Journal of Traditional and Complementary Medicine, 8(3):361-376.

Divya, S. and Sanjeev, S. (2021). Phytomedicine: Alternative safe vehicles on the pathway of diabetes mellitus. Ann. of Phytomed., 10(1): 114-122.

Kaleem, M.; Medha, P.; Ahmad, Q.U.; Asif, M. and Bano, B. (2008). Found pharmacological effects of Annona squamosa extract in Streptozotocin induced diabetic rats. Singapore Med. J., 49(10):800.

Latha, S.; Selvamani, P. and Prabha, T. (2021). Pharmacological Uses of the plants belonging to the genus commiphora. Cardiovasc. Hematol. Agents. Med. Chem., 19(2):101-117.

Mahendran, G.; Thamotharan, G; Sengottuvelu, S. and Narmatha Bai, V. (2014). Antidiabetic activity of Swertia corymbosa (Griseb.) Wight ex C.B. Clarke aerial parts extract in streptozotocin induced diabetic rats. Journal of Ethnopharmacology, 151(3)1175-1183.

Mohamed, B.; Abderrahim, Z.; Hassane, M.; Abdelhafid, T. and Abdelkhaleq, L. (2006). Medicinal plants with potential antidiabetic activity: A review of ten years of herbal. Int. J. Diabetes and Metabolism, 14: $1-25$. 
Nupur, M. (2021). Herbs that heal: Nature's pharmacy endowed remedies for better health. Ann. of Phytomed., 10(1):6-22

Petchi, R. R.; Vijaya, C. and Parasuraman, S. (2014). Antidiabetic activity of polyherbal formulation in streptozotocin: Nicotinamide induced diabetic wistar rats. Journal of Traditional and Complementary Medicine, 4(2): 108-117

Punit, R.B.; Kajal, B.P.; Urvesh, DP.; Chirag, M.M.; Harshad, B.P. and Bhavesh B.J. (2019). Antidiabetic, antioxidant and anti-inflammatory activity of medicinal plants collected from nearby area of Junagadh, Gujarat. Ann. of Phytomed., 8(2):75-84

Rang, H.P.; Dale, M.M. and Ritter, J.M. (2006). Pharmacology, New Delhi, Churchil living stone. $5^{\text {th }}$ Edition:380-393.

Sabu, M.C. and Ramadasan, K. (2009). Antidiabetic and antioxidant activity of Terminalia belerica, Roxb. Indian Journal of Experimental Biology, 47:270-275.
Satyanarayana, T.P.; Dwarakanadha, R.; Swarnalatha, D. and Anjana,A.M. (2010). Hypoglycemic effect of a polyherbal extract on normal and streptazotocin induced diabetic rats, International Journal of Pharmacy and Pharmaceutical Sciences, 2(3):56-57.

Shipra, G.; Suman, B.S.; Krishna, M.P. and Surendra, K.B. (2009). The protective role of Cassia auriculata leaf extract on hyperglycemia: Induced oxidative stress and its safety evaluation, Indian Journal Biochemistry and Biophysics. 46:371-377.

Singh, S.S.; Pandey, S.C.; Srivastava, S.; Gupta, V.S.; Patro, B. and Ghosh, A.C. (2003). Chemistry and medicinal properties of Tinospora cardifolia (Guduchi). Indian Journal of Pharmacology, 35:83-91.

Syed, M.A.; Vrushabendra, S.B.M.; Gopkumar, P.; Dhanapal, R. and Chandrashekara, V.M. (2005). Antidiabetic activity of Terminalia catappa Linn. leaf extracts in alloxan induced diabetic rats. Iranian Journal of Pharmacology and Therapeutics Copy Right, 4:36-39. 